\title{
Empyema editorial
}

\section{Daniel G. Dunlap ${ }^{1}$, Roy Semaan ${ }^{1}$, David Feller-Kopman ${ }^{2}$}

${ }^{1}$ Division of Pulmonary, Allergy and Critical Care Medicine, University of Pittsburgh Medical Center, Pittsburgh, PA, USA; ${ }^{2}$ Section of Interventional Pulmonology, Division of Pulmonary, Critical Care, and Sleep Medicine, Johns Hopkins Hospital, Baltimore, MD, USA

Correspondence to: David Feller-Kopman, MD. Director, Bronchoscopy \& Interventional Pulmonology, Professor of Medicine, Anesthesiology, Otolaryngology, Head and Neck Surgery, Pulmonary and Critical Care Medicine, Division of Pulmonary, Critical Care, and Sleep Medicine, Johns Hopkins Hospital, Sheikh Zayed Tower, Suite 7-125, 1800 Orleans Street, Baltimore, MD 21287, USA. Email: dfk@jhmi.edu.

Provenance: This is an invited Editorial commissioned by the Section Editor Laura Chiara Guglielmetti (Cantonal Hospital Winterthur, Kantonsspital Winterthur, Winterthur, Switzerland).

Comment on: Semenkovich TR, Olsen MA, Puri V, et al. Current State of Empyema Management. Ann Thorac Surg 2018;105:1589-96.

Submitted Aug 16, 2018. Accepted for publication Aug 23, 2018.

doi: $10.21037 /$ jtd.2018.08.135

View this article at: http://dx.doi.org/10.21037/jtd.2018.08.135

Empyema refers to a collection of frank pus or infected fluid within the pleural space and has an attributable mortality rate of $10-20 \%(1-3)$. The standard of care includes appropriate antibiotic therapy and prompt evacuation of the pleural fluid, as delay and a lack of source control leads to organization of the space making drainage difficult and significantly worsening outcomes (4). Though the importance of adequate empyema drainage was first recognized over 2,000 years ago (5), how best to accomplish this task remains controversial (6), with both primary chest tube insertion and surgical management routinely performed in clinical practice. Dr. Semenkovich and colleagues sought to investigate this treatment dilemma by performing a retrospective cohort study entitled "Current State of Empyema Management", in which the Healthcare Cost and Utilization Project New York State Inpatient Database [2009-2014] was queried for hospitalizations for empyema.

Once selected, 4,095 patients meeting eligibility criteria were sorted into three groups based upon the initial treatment they received during their first hospitalization for empyema: chest tube insertion, video-assisted thoracoscopic surgery (VATS), or open thoracotomy. The primary outcome was "treatment success," which the authors defined as: (I) management with a single procedure during index hospitalization; (II) no need for re-intervention within 30 days; and (III) patient survival through 30 days of followup. Initial chest tube insertion was performed in over twothirds of patients, but $44 \%$ of these individuals also had surgical management at some point during the course of their illness. Treatment success with initial chest tube insertion (without the need for additional procedures or surgery) was achieved in only $37 \%$ of patients as compared to $55 \%$ and $58 \%$ with initial VATS and open thoracotomy, respectively. Overall, a majority of patients in the study would eventually receive definitive surgical management, and 30-day mortality was significantly higher in the initial chest tube group when compared to patients undergoing primary VATS or open surgery $(13 \%$ vs. $5 \%$ and $6 \%$, respectively). Hospital length of stay was shortest in the initial VATS group (median 12 days) followed by initial chest tube and open thoracotomy groups (median 14 and 15 days, respectively). Interestingly, only $53 \%$ of all patients were treated with a single procedure or surgery, regardless of initial treatment modality. Notably, when this did occur, hospital length of stay was significantly shorter by an average of 3 days.

The strength of this study is its high number of participants, involving all hospitalized patients in the State of New York, which includes academic and private hospital centers of varying capabilities in both urban and rural areas. As a result, the authors are able to explore empyema management and outcomes in a broad range of clinical settings. However, we do have several concerns with this study mostly arising from its retrospective nature and the inability to control for severity of illness in the three cohorts. The stark contrast in patient characteristics between nonsurgical and surgical groups seems to go 
beyond what the authors concede to as "some degree of selection bias." Patients with primary chest tube insertion were almost a decade older than patients undergoing initial surgery, were more acutely ill, and were more frequently afflicted with sepsis (646 in the chest tube group $v s .442$ and 401 in respective surgical groups, $\mathrm{P}<0.001$ ) and shock (237 in the chest tube group vs. 103 and 123 in respective surgical groups, $\mathrm{P}<0.001)$. While it is certainly possible that the primary chest tube insertion group was sicker due to inadequate source control, another explanation is that these patients were deemed unstable to safely undergo surgery. As such, it is no surprise that their clinical outcomes were significantly worse. It is well known that elderly patients present in a more indolent fashion (typically with anemia, weight loss and failure to thrive) with a more complicated pleural space and a higher failure rate of non-surgical therapy (7).

In addition to being more acutely ill, patients that underwent initial chest tube insertion tended to be chronically ill, with significantly more medical comorbidities than the primary surgical cohorts, spanning almost every major organ system. Almost $10 \%$ of patients receiving an initial chest tube had actively metastatic cancer (compared to $2.8 \%$ and $4.3 \%$, respectively, in initial surgery groups) which one would expect to be associated with significantly worse outcomes regardless of treatment modality. The incidence of kidney disease, congestive heart failure, valvular issues, coagulopathy, and peripheral vascular disease were significantly higher in the nonsurgical cohort $(\mathrm{P}<0.001)$, all of which would make surgery a less attractive option.

Another limitation was the lack of data regarding intrapleural fibrinolytic therapies. Originally described in 1949 (8), fibrinolytic therapy can be instilled through a chest tube into the pleural space, thinning purulent material and disrupting loculations with the goal of promoting more robust drainage and achieving source control with parietal and visceral pleural apposition. It wasn't until the turn of the $21^{\text {st }}$ century that this practice was investigated. From 1997-2004 several small trials each examining around 50 patients or less comparing instillation of streptokinase or urokinase into chest drains versus surgery did not show a mortality difference, but did show a significant trend towards lower surgical referral rates (9-12). These smaller studies were followed by a large randomized controlled trial, MIST1, looking at streptokinase administered into the pleural space of 454 patients with empyema. Unfortunately, intrapleural streptokinase therapy did not demonstrate any improvement in mortality, rate of surgery, or length of hospital stay (1). However, in the subsequent MIST2 trial, a combination of tissue plasminogen-activator ( $\mathrm{tPA}$ ) and DNase (a thrombolytic and deoxyribonuclease, respectively) were instilled into the pleural space twice daily for three days (6 total doses) and compared to three other groups of thrombolytics alone, DNAase alone or placebo. Pleural combination therapy resulted in significantly improved opacity on chest imaging $(\mathrm{P}=0.005)$, shortened length of hospital stay, and decreased surgical referral at 3 months, though there was no difference in 3-month mortality (13). Neither tPA + placebo nor DNase + placebo therapies yielded significantly different results when compared to patients receiving double placebo. More recently, a retrospective cohort study suggested that combination intrapleural therapy was effective at completely evacuating the pleural space without the need for surgery $90 \%$ of the time, with almost $81 \%$ of patients effectively treated with fewer than six doses (median, 2) (14). As such, differentiating patients who received pleural combination therapy from those with lone chest tube insertion is a very important distinction which could not be evaluated in this study.

Finally, the authors' definition of "treatment success" is problematic. Dr. Semenkovich and colleagues recognize "the complexity of empyema management," as discussed towards the end of their manuscript, yet the criteria included in their primary outcome fails to adequately reflect this. Certainly, 30-day survival is an appropriate measure to include, but mandating that only one procedure or surgery be performed to achieve success fails to consider the many patients in whom a step-wise approach to therapy is sought, when multiple interventions are planned early in their course of illness. One such example would include patients with a very complex loculated pleural space in whom a chest drain is placed to instill tPA/DNase preoperatively to decrease loculations and improve drainage with the intention of increasing the likelihood of success with minimally invasive decortication using VATS without the need for thoracotomy. In such a cohort of patients, curative treatment without complications would be labeled as "treatment failure," which is misleading. Although an overall majority of patients required multiple interventions in general, "treatment success" seems to favor an early surgical approach by its very definition, rather than as a result of improved clinical outcomes.

Empyema management continues to be controversial and difficult due to the morbid procedures and surgeries 
required, as well as the septicemia that frequently ensues. Unfortunately, there appears to be no easy solution to this centuries-old problem. Indeed, early chest tube insertion with intrapleural fibrinolytic therapy does not appear to be the definitive answer, as this has not been proven to be superior to surgery (15). Perhaps the future of empyema care will include a combination of the two approaches, with chest tube drainage aided by tPA and DNase combination therapy for optimization prior to planned minimally invasive surgery. Further investigation of currently used and novel lytic agents with prospective, randomized-controlled trials is needed to guide initial therapy in order to improve outcomes. Until then, careful assessment of individual cases with a multi-disciplinary team including pulmonologists, thoracic surgeons, and intensivists seems the best way to ensure optimal care for this group of complex patients.

\section{Acknowledgements}

None.

\section{Footnote}

Conflicts of Interest: The authors have no conflicts of interest to declare.

\section{References}

1. Maskell NA, Davies CW, Nunn AJ, et al. U.K. Controlled trial of intrapleural streptokinase for pleural infection. $\mathrm{N}$ Engl J Med 2005;352:865-74.

2. Grijalva CG, Zhu Y, Nuorti JP, et al. Emergence of parapneumonic empyema in the USA. Thorax 2011;66:663-8.

3. Farjah F, Symons RG, Krishnadasan B, et al. Management of pleural space infections: a population-based analysis. J Thorac Cardiovasc Surg 2007;133:346-51.

4. Ashbaugh DG. Empyema thoracis. Factors influencing morbidity and mortality. Chest 1991;99:1162-5.

5. Adams F. The Genuine Works of Hippocrates. London: C. and J. Adlard Printers, 1849. Available online:

Cite this article as: Dunlap DG, Semaan R, Feller-Kopman D. Empyema editorial. J Thorac Dis 2018;10(Suppl 33):S3911S3913. doi: 10.21037/jtd.2018.08.135
https://books.google.com/books?id=OqAEAAAAQA AJ\&printsec=frontcover\&source=gbs_ge_summary $\mathrm{r} \& \mathrm{cad}=0 \# \mathrm{v}=$ onepage \&q\&f=false. Accessed 8/15/18.

6. Shen KR, Bribriesco A, Crabtree T, et al. The American Association for Thoracic Surgery consensus guidelines for the management of empyema. J Thorac Cardiovasc Surg 2017;153:e129-46.

7. El Solh AA, Alhajjhasan A, Ramadan FH, et al. A comparative study of community- and nursing home-acquired empyema thoracis. J Am Geriatr Soc 2007;55:1847-52.

8. TIllet WS, Sherry S. The effect in patients of streptococcal fibrinolysin (streptokinase) and streptococcal desoxyribonuclease on fibrinous, purulent, and sanguinous pleural exudations. J Clin Invest 1949;28:173-90.

9. Davies RJ, Traill ZC, Gleeson FV. Randomised controlled trial of intrapleural streptokinase in community acquired pleural infection. Thorax 1997;52:416-21.

10. Bouros D, Schiza S, Tzanakis N, et al. Intrapleural urokinase versus normal saline in the treatment of complicated parapneumonic effusions and empyema. A randomized, double-blind study. Am J Respir Crit Care Med 1999;159:37-42.

11. Tuncozgur B, Ustunsoy H, Sivrikoz MC, et al. Intrapleural urokinase in the management of parapneumonic empyema: a randomised controlled trial. Int J Clin Pract 2001;55:658-60.

12. Diacon AH, Theron J, Schuurmans MM, et al. Intrapleural streptokinase for empyema and complicated parapneumonic effusions. Am J Respir Crit Care Med 2004;170:49-53.

13. Rahman NM, Maskell NA, West A, et al. Intrapleural use of tissue plasminogen activator and DNase in pleural infection. N Engl J Med 2011;365:518-26.

14. Majid A, Kheir F, Folch A, et al. Concurrent Intrapleural Instillation of Tissue Plasminogen Activator and DNase for Pleural Infection. A Single-Center Experience. Ann Am Thorac Soc 2016;13:1512-8.

15. Redden MD, Chin TY, van Driel ML. Surgical versus non-surgical management for pleural empyema. Cochrane Database Syst Rev 2017;3:CD010651. 